
\title{
Research S Surate \\ ATG101 as a Novel Prognostic Biomarker Related to Immunotherapy in Pan-cancer
}

\section{Zijian Zhang}

Second Xiangya Hospital

Jinggang Mo

Taizhou University

Chong Jin

Taizhou University

Hao Jiang

Taizhou University

Zhongtao Liu

Second Xiangya Hospital

Heng Zou

Second Xiangya Hospital

Li Xiong

Second Xiangya Hospital

Chuang Teng

Taizhou University

Yu Wen

Second Xiangya Hospital

Kunpeng wang ( $\square$ wangkp9644@tzzxyy.com )

Taizhou University https://orcid.org/0000-0001-5088-2548

\section{Primary research}

Keywords: ATG101, pan-cancer, prognosis, immunotherapy

Posted Date: May 4th, 2021

DOI: https://doi.org/10.21203/rs.3.rs-430028/v1

License: (a) (i) This work is licensed under a Creative Commons Attribution 4.0 International License.

Read Full License 


\section{Abstract}

Background: ATG101 plays a significant role in the occurrence and development of tumours by regulating autophagy. Our study aimed to research the correlation between the expression of ATG101 and tumour prognosis and its role in tumour immunity.

Methods: First, integrated analysis of The Cancer Genome Atlas and Genotype-Tissue Expression portals were used to analyse the expression of ATG101. Then, we used Kaplan-Meier curves for survival analysis. Next, we analysed the relationship between ATG101 expression and six immune cells, the immune microenvironment and immune checkpoints. Besides, we analysed the relationship between the expression of ATG101 and methyltransferase. Finally, we used GSEA to study the function of ATG101 in COAD and LIHC.

Results: Integrated analysis showed that ATG101 was overexpressed in different tumours. Kaplan-Meier curves found that ATG101 was associated with poor prognosis in most tumours. We found that that ATG101 can be used as a target and prognostic marker of tumour immunotherapy for different tumours. We also found that ATG101 regulates DNA methylation. GSEA analysis showed that ATG101 may play a critical role in COAD and LIHC.

Conclusions: Our study highlights the significance of ATG101 in the study of tumour immunity from a pan-cancer perspective.

\section{Background}

Currently, cancer is still a serious health burden; over 19.3 million new cases and nearly 10.0 million deaths were estimated for 36 cancers across 185 countries in $2020^{[1]}$. The four leading causes of cancerrelated deaths were lung, colorectal, liver and stomach cancer. Breast cancer and lung cancer were the most commonly diagnosed cancers in females and males, respectively ${ }^{[1]}$. The increasing incidence of cancer places a heavy burden on both developed and developing countries ${ }^{[2]}$. Surgical resection, radiotherapy, chemotherapy and ablation therapy (including photodynamic therapy, microwave ablation and radiofrequency ablation) are typical therapies for cancer treatment ${ }^{[3]}$, but their efficacy is still limited owing to delayed cancer detection and tumour heterogeneity ${ }^{[4]}$. Mounting evidence indicates that immunotherapy, including immune checkpoint blockade, is a potential alternative therapy for curing various cancers $^{[5,6]}$. ATG101 is not only a critical factor for autophagy but also a potential therapeutic target for immunotherapy ${ }^{[7]}$. However, there are no studies on the significance of the association between ATG101 and the immune microenvironment across cancers.

Here, we first predicted the function of ATG101 in the prognosis of immunotherapy associated with various cancers via bioinformatics techniques. Moreover, we analyzed the relationship between ATG101 and forty-seven common immune checkpoint genes as well as the relationship of ATG101 expression levels with the expression of methyltransferases in various cancers. Finally, we explained the regulatory 
mechanism of ATG101 in different cancers by GSEA enrichment analysis. Our results provide the first strong evidence for ATG101 as a target for immunotherapy from the perspective of pan-cancer and suggest that ATG101 can be used as a prognostic marker for immunotherapy.

\section{Methods}

\section{Obtain ATG101 expression data from public databases}

The expression of ATG101 in various normal tissues and cell lines was analyzed using data from the GTEx and CCLE databases. Data from a total of 6678 normal tissues and organs were obtained from the GTEx database (https://www.gtexp ortal.org/home/). We collected 11057 samples from the TCGA database, including 10327 tumour samples and 730 paraneoplastic samples matched with 33 types of malignant cancers. For the calculation of the difference in ATG101 expression between tumour and paraneoplastic tissues, some tumours with missing matches or too few paraneoplastic samples were excluded. For the remaining samples, the tumour samples were normalized and then subjected to the Wilcoxon test to analyze whether there were differences in ATG101 expression between these cancers and normal tissues.

\section{Correlation between the expression of ATG101 and the prognostic value in various cancer patients}

To verify whether the expression of ATG101 significantly affects the prognosis of tumours, the survival information (including the OS, DSS, DFS and PFS rates) of the patients from whom the 10327 tumour samples from 33 cancer types in the TCGA database were obtained was downloaded. The Kaplan-Meier survival estimation method was used to calculate the prognostic value of ATG101, and the log-rank test was used to determine the significant difference index. With $\mathrm{P}<0.05$ indicating a significant difference, the results obtained by these two methods were analyzed.

\section{Correlation analysis between immune cell infiltration and ATG101 expression}

Through the TIMER algorithm, we used the gene expression profiles of 10897 samples from 32 cancer types in the TCGA database to infer the number of tumour-infiltrating immune cells (TIICs). TIMER is a statistical method of deconvolution that can be used for approximate calculation of immune infiltration. We analyzed the relationship between the expression level of the ATG101 gene and the abundance of infiltrating immune cells (including $\mathrm{CD} 4^{+} \mathrm{T}$ cells, $\mathrm{CD} 8^{+} \mathrm{T}$ cells, $\mathrm{B}$ cells, neutrophils, dendritic cells, and macrophages) through the above methods. 


\section{Correlation analysis between the expression level of ATG101 and the tumour microenvironment}

The non-tumour component cells in the tumour microenvironment, including immune cells and stromal cells, are mainly used for cancer diagnosis and prognostic evaluation. ESTIMATE (https://bioinformatics.mdanderson.org/public-software/estimate/) is a commonly used tool to predict the infiltration of stromal cells and immune cells in the tumour microenvironment and the purity of cancer tissues. According to the expression data from 9664 samples of 33 malignant cancer types, after excluding the normal tissue data, the ratio of stromal cells and immune cells in each tumour sample was calculated, and then the immune score, stromal score and ESTIMATE score were used to evaluate tumour purity. Furthermore, the ATG101 expression data and the score of the estimation algorithm were combined, and the Spearman correlation test was used to verify their correlation. This process mainly involved the following packages: ggplot2 (https://CRAN.R-project.org/package=ggplot2), ggpubr and ggExtra (https://CRAN. R-proje ct.org/packa ge = ggExtra).

\section{The correlation of TMB, MSI and neoantigens with the expression of ATG101}

Tumour mutation burden (TMB) and microsatellite instability (MSI) are important evaluation indicators directly related to the effect of immunotherapy, such as PD-1 blockade. The mutation data from 10114 samples from 33 cancer types were used to analyze the correlation between TMB and ATG101 expression levels. First, we calculated the mutation score of each tumour sample and obtained the TMB information for each cancer. In addition, we used the Spearman correlation test to analyze the correlation between ATG101 expression and TMB and used the fmsb software package (https://CRAN.Rproject.org/package $=\mathrm{fmsb}$ ) to construct a related radar chart. We also downloaded and analyzed the MSI scores of 10,415 tumour samples and used the same processing method to draw related radar charts of ATG101 expression and MSI. Predicted neoantigens of each sample were retrieved from a previous publication $^{[8]}$. The correlations between TMB, MSI, neoantigens and ATG101 expression were all calculated using the Spearman correlation test.

\section{The correlation between ATG101 and known essential marker genes}

Considering that the analysis results are intended to guide clinical practice, after exploring the relationship between ATG101 expressoin and immune cell infiltration, the correlation between ATG101 expression and various (47) immune checkpoint target genes was further analyzed. Here, the Spearman correlation test was used. Next, the reshape2 software package (http://www.jstatsoft.org/v21/i12/) was used to establish a related heat map. Changes in the DNA methylation state are common in tumours. 
DNA methylation plays an important role in the occurrence and development of tumours. Therefore, we deeply analyzed the correlation between four methyltransferases (DNMT1, DNMT2, DNMT3A and DNMT3B) and the expression level of ATG101. The analysis method was the same as that described above.

\section{Gene Set Enrichment Analysis (GSEA) analysis}

ATG101 expression is closely related to patient prognosis and immune regulation responsiveness in COAD and LIHC; thus, we aimed to determine the potential mechanisms and signalling pathways of ATG101 in different tumours. First, according to the expression level of ATG101, the tumours were divided into high expression groups and low expression groups. Then, limma, org.Hs.eg.db, clusterProfiler (http://bioconductor.org/packages/relea se/bioc/html/clust erPro filer.html) and c2.cp.kegg.v7.2.symbols background were used to perform gene set enrichment analysis on tumours and to select the most important KEGG pathway to draw an enrichment curve. $P<0.05$ was used as the standard of significant difference. Then, the transcription factor information related to ATG101 binding was downloaded from ChIPBase, and the genes enriched by GSEA intersected with the genes identified in ChIPBase. Twenty-one common genes were obtained. These 21 genes were then analyzed by STRING (https://www.string$\mathrm{db}$. org/)) and visualised with Cytoscape

\section{Statistical analysis}

All data in this study were analyzed using R version 4.0.3 (https://www.r-project.org/) and its auxiliary software packages, and all analyses were performed with $\mathrm{P}<0.05$ as the standard of significant difference. When analyzing the expression differences of ATG101, the Limma software package and Student's t-test were used, the Kaplan-Meier curve was used for survival analysis, and the Spearman or part of the Spearman method was used to analyze the correlation between different genes and the relationship between different genes and different immune cells.

\section{Results}

\section{Expression of ATG101 in pan-cancer}

First, we compared tumour samples and paracarcinoma tissues from the TCGA database, normal samples from the GTEx database and cancer cells from the CCLE database to evaluate the mRNA expression characteristics of ATG101 in humans. We found that the expression of ATG101 varied in different normal tissues (Figure 1A) and cancer cells (Figure 1B) after collating the tumour tissues and paracarcinoma tissues from the TCGA database. We found that the expression of ATG101 was upregulated in BRCA, CHOL, COAD, ESCA, HNSC, LIHC, LUAD, LUSC, PRAD, READ, STAD, and UCEC. ATG101 was downregulated only in KIRP (Figure 1C). Then, we analyzed the difference in ATG101 expression between normal samples from the GTEx database and tumour tissues from the TGGA 
database and found that ATG101 expression was upregulated in BRCA, CESC, CHOL, COAD, GBM, HNSC, $\mathrm{KICH}, \mathrm{LGG}$, LIHC, LUSC, OV, PAAD, PRAD, READ, SKCM, UCEC and UCS. However, its expression was found to be downregulated in ESCA, LAML, LUAD, SKCM, STAD and TGCT (Figure 1D).

\section{Correlation analysis between ATG101 expression level and prognostic value}

The characteristics of ATG101 expression at the mRNA level suggested that ATG101 may be a valuable target for pan-cancer. Therefore, we further used Kaplan-Meier analysis to explore the correlation between ATG101 mRNA expression levels and the survival outcomes (including the OS, DSS, DFS and PFS rates) of different cancers from the TCGA database. Our results demonstrated that upregulated ATG101 expression was associated with a shorter OS rate in ACC, CHOL, LGG, LIHC and MESO (Figure 2 A-E). In addition, upregulation of ATG101 expression was related to a shorter DSS rate in ACC, COAD, KIRP, LGG, LIHC, MESO and READ (Figure 2 F-L). High ATG101 expression was associated with a poor DFS rate in ACC, CHOL and LUSC, while low ATG101 expression was associated with a poor DFS rate in USC (Figure $2 \mathrm{M}-\mathrm{P}$ ). Furthermore, the high expression of ATG101 was also associated with a poor PFS rate in ACC, COAD, KIRP and LIHC (Figure $2 \mathrm{Q}-\mathrm{T}$ ). These results confirmed that the high expression of ATG101 is negatively correlated with the prognosis of most malignant tumours.

\section{Correlation analysis between ATG101 expression and immune cells}

We explored the relationship between ATG101 expression and immune infiltration in different tumours after investigating the relationship between ATG101 expression and cancer prognosis. An analysis of six tumour-infiltrating immune cells (B cells, CD4+ T cells, CD8+ T cells, neutrophils, macrophages and dendritic cells) was conducted. With the cut-off of $p$ value of 0.05 , ATG101 expression was positively correlated with tumour-infiltrating immune cells. In LIHC (Figure 3 A), ATG101 expression was positively correlated with $B$ cells (correlation coefficient $=0.37$, $p$ value $=1.4 \mathrm{e}-13$ ), $C D 4+T$ cells (correlation coefficient $=0.313, p$ value $=6.31 \mathrm{e}-10), \mathrm{CD} 8+\mathrm{T}$ cells (correlation coefficient $=0.284, \mathrm{p}$ value $=2.5 \mathrm{e}-08$ ), dendritic cells (correlation coefficient $=0.416$, $p$ value $=4.5 \mathrm{e}-17$ ), macrophages (correlation coefficient $=0.407, p$ value $=2.75 e-16$ ) and neutrophils (correlation coefficient $=0.398, p$ value $=1.31 \mathrm{e}-15$ ). In LUSC (Figure 3 B), ATG101 expression was positively correlated with B cells (correlation coefficient $=0.098, p$ value $=0.0284), C D 4+T$ cells (correlation coefficient $=0.146, p$ value $=0.00107), C D 8+T$ cells (correlation coefficient $=0.142$, $p$ value $=0.00149$ ), dendritic cells (correlation coefficient $=0.152, p$ value $=0.000633$ ), macrophages (correlation coefficient $=0.156, p$ value $=0.000472$ ) and neutrophils (correlation coefficient $=0.214$, p value $=1.34$-06). In PCPG (Figure 3 C), ATG101 expression was positively correlated with $B$ cells (correlation coefficient $=0.295$, $p$ value $=5.31 \mathrm{e}-09$ ), $C D 4+T$ cells (correlation coefficient $=0.243$, $p$ value $=0.000931), C D 8+T$ cells (correlation coefficient $=0.164, p$ value $=0.0265$ ), 


\section{Relationship between ATG101 expression and the tumour immune microenvironment}

Based on the correlation between immune cells and ATG101 expression, we also explored the relationship between ATG101 expression and the tumour immune microenvironment. Our results showed that ATG101 expression was significantly positively correlated with the immune score in BLCA, BRCA, CESC, COAD, DLBC, ESCA, GBM, HNSC, KICH, KIRC, LAML, LGG, LIHC, LUAD, LUSC, PCPG, SARC and UVM (Figure S1). ATG101 expression was significantly positively correlated with stromal score in BLCA, BRCA, CESC, COAD, GBM, HNSC, KICH, KIRP, LGG, LIHC, LUAD, LUSC, MESO, PCPG, PRAD, SARC, STAD, TGCT, THCA, THYM, UCEC and UVM (Figure S2). Moreover, ATG101 expression was significantly positively correlated with the ESTIMATE score in BLCA, BRCA, CESC, COAD, ESCA, GBM, HNSC, KICH, LAML, LGG, LIHC, LUAD, LUSC, MESO, PCPG, SARC and UVM (Figure S3). ATG101 expression was found to be significantly positively correlated with all three scores in BLCA, BRCA, CESC, COAD, GBM, HNSC, KICH, LGG, LIHC, LUAD, LUSC, PCPG, SARC and UVM. Overall, ATG101 expression had the third highest correlation with stromal score in LUSC $(r=-0.212, P<0.001)$, TGCT $(r=-0.353, P<0.001)$, and PCPG $(r=0.315, P<0.001)$. ATG101 expression had the third highest correlation with immune score in LUAD $(r=-0.174, P<0.001), \operatorname{LUSC}(r=$ $-0.212, P<0.001)$, HNSC $(r=-0.142, P<0.001)$ and the third highest correlation with the ESTIMATE score in LUSC $(r=-0.212, P<0.001)$, LUAD $(r=-0.174, P<0.001)$, PCPG $(r=0.315, P<0.001)$ (Figure 4).

\section{Relationship between ATG101 expression and immune checkpoint gene expression in various cancers}

The immune checkpoint is an inhibitory signalling pathway in the immune system that regulates the intensity and persistence of the immune response in peripheral tissue, prevents tissue injury and plays an important role in maintaining self-antigen tolerance. We used the mRNA sequence database to assess whether there was an association between the expression level of ATG101 and 47 common immune checkpoint genes. We found that ATG101 was highly correlated with immune checkpoint genes in various cancers. In BRCA, COAD, LIHC, LUAD and PCPG, we found that ATG101 and other immune checkpoint genes were significantly coexpressed. However, the expression of ATG101 was negatively correlated with all forty-seven immune checkpoint genes in CHOL, DCBC, TGCT and UCS (Figure 5 A).

\section{Relationship between ATG101 expression and TMB and MSI in various cancers}


Classic immune checkpoint therapeutic targets, including PD-1, PD-L1 and CTLA-4, are widely targeted clinically and these therapies have achieved satisfactory effects. TMB and MSI are important evaluation indexes directly related to the efficacy of immune checkpoint therapies such as PD-1 blockade. We found that TMB was significantly correlated with the expression levels of ATG101 in COAD, HNSC, LGG, LUAD, SARC, SKCM, STAD, THCA and THYM $(P<0.05)$. In addition, UCEC had the lowest TMB score, and SKCM had the highest. (Figure $5 \mathrm{~B}$ ). The results showed that ATG101 expression is negatively correlated with the hypermutation state in UCE but positively correlated with the hypermutation state in SKCM. We also researched the correlation between ATG101 expression and MSI in various cancers. We found that ATG101 expression was significantly correlated with MSI in COAD, DLBC, HNSC, KICH, KIRC, KIRP, READ, SARC and SKCM $(P<0.05)$, while DLBC had the highest coefficient and CHOL had the lowest score (Figure $5 \mathrm{C})$.

\section{Relationship between ATG101 expression and neoantigens in various cancers}

Neoantigens are new antigens produced by tumour cells after tumour mutation and are different from those expressed by normal cells. The more neoantigens are produced, the easier it is for tumour cells to be recognized and attacked by immune cells, which is more conducive to the development of new targeted drugs. The development of bioinformatics technology has accelerated the identification of neoantigens. Here, we further analyzed the relationship between ATG101 expression and pan-cancer neoantigens to provide a reference for new immunotherapies for tumours. Our results showed that ATG101 expression was positively correlated with neoantigens in BRCA, KIRC, READ, HNSC, LIHC, SKCM and CESC $(P<0.05)$. However, in GBM, OV, LUAD, LUSC, KIRP, UCEC, COAD, STAD, THCA, BLCA, PRAD and LGG, ATG101 expression was negatively correlated with neoantigens (Figure S4).

\section{Relationship between ATG101 expression and the expression of four methyltransferases in various cancers}

DNA methylation is a physiological process that changes chromatin structure, DNA conformation, DNA stability, and the interaction between DNA and protein through the action of DNA methyltransferase. It is one of the main mechanisms by which gene expression is regulated. DNA methylation is considered to be one of the main factors affecting tumour occurrence and development. In our research, we explored the correlation between ATG101 expression and the expression of four methyltransferases (DNMT1, DNMT2, DNMT3A and DNMT3B) (Figure 6). In STAD, THCA, THYM, UCEC, UVM, ACC, BLCA, BRCA, CESC, CHOL, COAD, DLBC, ESCA, GBM, HNSC, KICH, KIRC, KIRP, LGG, LIHC, LUAD, LUSC, MESO, OV, PAAD, PCPG, PEAD and SKCM, ATG101 expression was positively correlated with DNA methylation $(P<0.05)$. In contrast, ATG101 expression was negatively correlated with DNA methylation in UCS, SARC, and TGCT $(P<0.05)$. 


\section{Gene set enrichment analysis of ATG101 in COAD and LIHC}

After preliminary exploration of the genetic and epigenetic mechanisms of ATG101, we used GSEA to research the function of ATG101 in COAD and LIHC. In COAD, ATG101 can inhibit proteasome, RNA polymerase, BASE excision repair, DNA replication, and ribosome (Figure $7 \mathrm{~A}$ ), but promote Wint signaling pathway, ERBB signaling pathway, Adherens junction, and aldosterone regulated sodium reabsorption (Figure 7 B). While in LIHC, ATG101 can inhibit Base excision repair, spliceosome, RNA degradation, cytosolic DNA sensing pathway, and epitheliak cell signaling in helicobacter pylor (Figure 7 C), but promote complement and coagulation cascades, butanoate metabolism, valine leucine and isoleucine degradation, drug metabolism cytochrome P450, and fatty acid metabolism (Figure 7 D). As for transcription factor level, ATG101 can promote the function of transcription factor 4, androgen receptor, signal transducer and activator of transcription 5A and $Y Y 1$ transcription factor (Figure $7 \mathrm{E}$ ). In addition, ATG101 can inhibit the function of members of the ETS oncogene family, v-myc avian myelocytomatosis viral oncogene homologue, cAMP responsive element binding protein 1 , v-ets avian erythroblastosis virus E26 oncogene homologue and early growth response 1 (Figure 7 F). In LIHC, ATG101 can affect the function of v-myc avian myelocytomatosis viral oncogene homologue, GA binding protein transcription factor alpha subunit, ETS oncogene family, v-myc avian myelocytomatosis viral oncogene homologue and v-myc avian myelocytomatosis viral oncogene homologue (Figure $7 \mathrm{G}$ ). The transcription factor binding information for ATG101 was then downloaded from ChIPBase, and the genes enriched by GSEA were intersected with the genes identified from ChIPBase. Twenty-one common genes related to ATG101, COAD and LIHC were obtained (figure $7 \mathrm{H}$ ).

\section{Discussion}

ATG101, a novel mammalian autophagy factor thought to directly interact with Atg13, was first identified by Hosokawa in $2009^{[9]}$. Previous studies regarding ATG101 were mainly in the field of autophagy. ATG101 is an essential gene for the initiation of autophagy and might be a potential therapeutic target in diseases involving endothelial injury ${ }^{[7]}$. Meanwhile, ATG101 also functions together with FIP200 ATG13 and $U \mathrm{LK}^{[9]}$. Regulating ULK1 to regulate autophagy/autophagy-related cell death (ACD) may be effective in the treatment of triple-negative breast cancer ${ }^{[10]}$. Moreover, ATG101 is essential for tissue homeostasis in both adult brains and midguts ${ }^{[11]}$ and mediates GSN and OAS2, which are positively and negatively associated with the recurrence of colorectal cancer, respectively ${ }^{[12]}$. Some scholars have used 7 ATGrelated genes to establish the prognostic risk characteristics of hepatocellular carcinoma ${ }^{[13]}$. Another previous study showed that stable knockdown of ATG5 can significantly inhibit the occurrence and progression of colorectal cancer tumours in vivo ${ }^{[14]}$. In addition, ATG genes are mainly associated with the regulatory mechanism of cell-specific gene expression in cholangiocarcinoma ${ }^{[15]}$. However, frameshift mutations of ATG-related genes may affect tumour progression by regulating autophagy in gastric cancer and colorectal cancer ${ }^{[16]}$. These studies are consistent with the results of our study. Therefore, ATG101 is expected to be a diagnostic and prognostic marker of different tumours. However, there are no studies on 
the significance of ATG101 in pan-cancer or its role in the immune microenvironment. In this study, we explored for the first time the relationship between ATG101 and different tumours from a pan-cancer perspective.

Cancer neoantigens are generally considered to be a new class of antigens produced as a result of individual cancer cell mutations. Because of their immunogenicity and low expression in normal tissues, cancer neoantigens are considered to be an important target for cancer immunotherapy. ${ }^{[17]}$. Changes in the abundance and mutation profile of ATG101 in various cancers leads to the emergence of new antigenic epitopes. Some initial, authoritative clinical trials revealed that dendritic cell-related neoantigen vaccines are safe and can induce CD8+ and CD4+ neoantigen-specific $T$ cell responses, which have broad application prospects ${ }^{[18]}$. In fact, dendritic cells (DCs) are an important part of the immune microenvironment. An increasing number of studies have revealed that DC vaccines with specific genes (such as ATG101) can effectively initiate adaptive cytolytic immune responses. For example, one prospective study confirmed the safety and effectiveness of vaccines against cancer neoantigens. The cancer neoantigen vaccine can induce specific CD4+ and CD8+ T cells to target specific patient tumours, and in further clinical trials, it is expected that the effect of the vaccine will be satisfactory ${ }^{[19]}$. In addition, Beatriz confirmed in patients with advanced melanoma that the DC vaccine can improve naturally occurring neoantigen-specific immunity and discovered a new human leukocyte antigen (HLA) neoantigen ${ }^{[20]}$. In addition, there is an urgent need to develop vaccines that target DCs or use them to present antitumour antigens in clinical practice ${ }^{[21]}$.

In this study, we attempted to deeply explore the relationship between immunity and ATG101 expression; therefore, we analysed the correlation between six types of immune cells, B cells, CD4+ T cells, CD8+ T cells, neutrophils, macrophages and dendritic cells, and the expression levels of ATG101. We found that in LIHC, LUSC and PCPG, the expression of ATG101 was positively correlated with all six immune cells. ATG101 may be a potential target for immunotherapy of these cancers. We further studied the relationship between ATG101 expression and neoantigens in various cancers. Our results showed that ATG101 expression was positively correlated with neoantigens in BRCA, KIRC, READ, HNSC, LIHC, SKCM and CESC. LIHC (liver hepatocellular carcinoma) was the only cancer in which there was a positive correlation among all six immune cells and neoantigens. The LHIC tumour microenvironment is complex and changeable. The TME affects the process of cancer cell antigen presentation by expressing tumour antigens, thereby allowing tumour cells to evade effective tumour treatment methods ${ }^{[22]}$. The complex tumour immune microenvironment of the LHIC is also one of the main causes of the heterogeneity of the treatment response to immune checkpoint blockers such as PD-1 and CTLA-4 blockade in LHIC patients with the same TNM stage ${ }^{[23]}$. We also studied the relationship between ATG101 expression and immune checkpoint genes. In BRCA, COAD, LIHC, LUAD and PCPG, we found that ATG101 and other immune checkpoint genes were significantly coexpressed. However, the expression of ATG101 was negatively correlated with all forty-seven immune checkpoint genes in CHOL, DCBC, TGCT and UCS. Therefore, ATG101 may be a potential biomarker to determine the prognosis of LHIC patients. 
TMB is the total number of mutations per megabase in cancer, representing tumour mutation quantity. The more tumour mutations there are, the greater the differences between the cancer cells and normal cells. That is, the higher the value of TMB is, the more likely the cancer is to be recognized and attacked by the immune system and the better the response to immunotherapy. In recent years, TMB has been considered a biomarker for immunotherapy and an important evaluation index directly related to the efficacy of immune checkpoint therapy, such as PD-1 and CTLA-4 blockade ${ }^{[24]}$. Similarly, the formation of MSI caused by DNA MMR protein defects results in the accumulation of mutations and the production of neoantigens. MSI is also an important marker that determines the effectiveness of immunotherapy ${ }^{[25]}$. Our results showed that ATG101 expression was significantly correlated with both MSI and TMB only in COAD, HNSC and SKCM. This indicates that there is a significant negative correlation between ATG101 expression and $\mathrm{MSI}$ and TMB in these cancers. Our results are consistent with the results of previous studies. Maria demonstrated that immunogenic ileal apoptosis contributes to the prognosis of chemotherapy-treated $\operatorname{COAD}^{[26]}$. In addition, Simone's research showed that $\mathrm{CD} 8+\mathrm{T}$ cell-related therapies can be used for anticancer immunotherapy for $\mathrm{SKCM}^{[27]}$. Similar results have also been verified in HNSC $^{[28]}$.

In recent years, an increasing number of studies have shown that DNA methylation is highly related to the diagnosis, prognosis and prediction of the response to therapies for tumours, such as NSCL, colorectal cancer, hepatocellular carcinoma and metastatic breast cancer ${ }^{[29-31]}$. DNA methylation is one of the most common epigenetic events in the mammalian genome. Normal DNA methylation can maintain the normal functions of the body, such as the stability of the genome structure, normal embryonic development and cell differentiation, while abnormal DNA methylation will lead to tumorigenesis ${ }^{[32]}$. Some studies have indicated that the ATG gene may be involved in the development and progression of various diseases through promoting demethylation, regulating a variety of cellular functions and signalling pathways ${ }^{[33-35]}$. These studies indicated that DNA methylation may play a critical role in ATG gene expression. This study also revealed that the expression of several DNA methylation/demethylation factors has a significant correlation with ATG101 methylation status.

In this study, we found that the correlation between the expression levels of DNA methyltransferase and ATG101 varied in different tumours. Moreover, we explored the function of ATG101 in COAD and LIHC. Twenty-one common genes related to ATG101, COAD and LIHC were obtained. Our results are in agreement with previous studies. It was concluded that targeting STAT5-and STAT6-related signalling pathways can inhibit the proliferation of CRC cells and induce CRC cell apoptosis ${ }^{[36]}$, and Dong suggested that STAT5A, STAT5B and STAT6 expression may be potential prognostic markers of hepatocellular carcinoma ${ }^{[37]}$. MiR-532-3p can inhibit the progression of colorectal cancer by regulating ETS1-related signalling pathways ${ }^{[38]}$. Meanwhile, WTAP plays an important role in the progression of hepatocellular carcinoma by affecting the epigenetic modifications of ETS1 ${ }^{[39]}$. Chen concluded that MJD1C can affect colorectal cancer metastasis by targeting ATF2 ${ }^{[40]}$. However, further research is still needed to explore ATG101 epigenetic changes and its potential functions, which may contribute to the 
development of new cancer treatments and the discovery of new markers to predict the prognosis of patients with cancer.

\section{Conclusions}

In summary, our study is the first to demonstrate the relationship between the expression of ATG101 and tumour prognosis and the role of ATG101 in tumour immunity. Our research highlights the significance of ATG101 in the study of tumour immunity from the perspective of pan-cancer. However, our results need to be further verified in large-sample genome sequencing and in vivo and in vitro functional experiments.

\section{Abbreviations}

TCGA: The Cancer Genome Atlas; GEO: Gene Expression Omnibus; CCLE: Cancer Cell Line Encyclopedia; TMB: tumor mutation burden; MSI: microsatellite instability; OS: Overall survival ; DFS: disease free survival; PFS: progression free survival; DSS: disease special survival; ACC: Adrenocortical carcinoma; BLCA: Bladder Urothelial Carcinoma; BRCA: Breast invasive carcinoma; CESC: Cervical squamous cell carcinoma and endocervical adenocarcinoma; $\mathrm{CHOL}$ : Cholangiocarcinoma; COAD: Colon adenocarcinoma; READ: Rectum adenocarcinoma Esophageal carcinoma; DLBC: Lymphoid Neoplasm Diffuse Large B-cell Lymphoma; ESCA: Esophageal carcinoma; FPPP FFPE Pilot Phase II FFPE; GBM: Glioblastoma multiforme; GBMLGG: Glioma; HNSC: Head and Neck squamous cell carcinoma; KICH: Kidney Chromophobe; KIPAN: Pan-kidney cohort (KICH+KIRC+KIRP) ; KIRC: Kidney renal clear cell carcinoma; KIRP: Kidney renal papillary cell carcinoma; LAML: Acute Myeloid Leukemia; LGG :Brain Lower Grade Glioma; LIHC: Liver hepatocellular carcinoma; LUAD Lung adenocarcinoma; LUSC: Lung squamous cell carcinoma; MESO: Mesothelioma; OV: Ovarian serous cystadenocarcinoma; PAAD: Pancreatic adenocarcinoma; PCPG: Pheochromocytoma and Paraganglioma; PRAD: Prostate adenocarcinoma; READ: Rectum adenocarcinoma; SARC: Sarcoma; SKCM: Skin Cutaneous Melanoma; STAD: Stomach adenocarcinoma; STES: Stomach and Esophageal carcinoma; TGCT: Testicular Germ Cell Tumors; THCA: Thyroid carcinoma; THYM: Thymoma; UCEC: Uterine Corpus Endometrial Carcinoma; UCS: Uterine Carcinosarcoma; UVM: Uveal Melanoma

\section{Declarations}

\section{Ethics approval and consent to participate}

Not applicable.

\section{Consent for publication}

Not applicable. 


\section{Availability of data and materials}

Publicly available datasets were analyzed in this study; these can be found in The Cancer Genome Atlas (TCGA) (https://portal.gdc.cancer.gov/), GTEx database (https://www.gtexp ortal.org/home/) and GSE14520 (https://www.ncbi.nlm.nih.gov/geo/).

\section{Competing interests}

Not applicable.

\section{Funding}

This work was supported by the Zhejiang Province Public Welfare Technology Application Research Project (LGF21H160022), Zhejiang Medical and Health Science and Technology Program (2017KY711), Project of Taizhou Central Hospital (2019KT003), Project of Taizhou University (2018PY057), National Natural Science Foundation of China, No. 81970569, No. 81773293; Natural Science Foundation of Hunan Province, No. 2017 SK50121.

\section{Authors' contributions}

ZZJ, TC, WY, MJG, JC, and WKP contributed to data analysis, interpretation, and drafting. TC, WY, LZT, XL, $\mathrm{JH}, \mathrm{ZZJ}$, and WKP contributed to study design, study supervision, and final approval of the manuscript. All authors read and approved the final manuscript.

\section{Acknowledgements}

Not applicable.

\section{References}

1. Sung $H$, Ferlay J, Siegel R L, Laversanne M, Soerjomataram I, Jemal A囚 et al. Global cancer statistics 2020: GLOBOCAN estimates of incidence and mortality worldwide for 36 cancers in 185 countries[J]. CA Cancer J Clin, 2021.

2. Ouyang G, Liu Q, Wu Y, Liu Z, Lu W, Li S, et al. The global, regional, and national burden of gallbladder and biliary tract cancer and its attributable risk factors in 195 countries and territories, 1990 to 2017: a systematic analysis for the Global Burden of Disease Study 2017[J]. Cancer, 2021.

3. Kaibori M, Kosaka H, Matsui K, Ishizaki M, Matsushima H, Tsuda T, et al. Near-Infrared Fluorescence Imaging and Photodynamic Therapy for Liver Tumors[J]. Front Oncol, 2021,11:638327. 
4. Kong F, Zou H, Liu X, He J, Zheng Y, Xiong L, et al. miR-7112-3p targets PERK to regulate the endoplasmic reticulum stress pathway and apoptosis induced by photodynamic therapy in colorectal cancer CX-1 cells[J]. Photodiagnosis Photodyn Ther, 2020,29:101663.

5. de Gooijer C J, Borm F J, Scherpereel A, Baas P. Immunotherapy in Malignant Pleural Mesothelioma[J]. Front Oncol, 2020,10:187.

6. O'Donnell J S, Teng M, Smyth M J. Cancer immunoediting and resistance to T cell-based immunotherapy[J]. Nat Rev Clin Oncol, 2019,16(3):151-167.

7. Du J, Xu Z, Liu Q, Yang Y, Qian H, Hu M, et al. ATG101 Single-Stranded Antisense RNA-Loaded Triangular DNA Nanoparticles Control Human Pulmonary Endothelial Growth via Regulation of Cell Macroautophagy[J]. ACS Appl Mater Interfaces, 2017,9(49):42544-42555.

8. Rooney M S, Shukla S A, Wu C J, Getz G, Hacohen N. Molecular and genetic properties of tumors associated with local immune cytolytic activity[J]. Cell, 2015,160(1-2):48-61.

9. Hosokawa N, Sasaki T, lemura S, Natsume T, Hara T, Mizushima N. Atg101, a novel mammalian autophagy protein interacting with Atg13[J]. Autophagy, 2009,5(7):973-979.

10. Ouyang L, Zhang L, Fu L, Liu B. A small-molecule activator induces ULK1-modulating autophagyassociated cell death in triple negative breast cancer[J]. Autophagy, 2017,13(4):777-778.

11. Guo T, Nan Z, Miao C, Jin X, Yang W, Wang Z, et al. The autophagy-related gene Atg101 in Drosophila regulates both neuron and midgut homeostasis[J]. J Biol Chem, 2019,294(14):5666-5676.

12. Kim J C, Ha Y J, Tak K H, Roh S A, Kwon Y H, Kim C W, et al. Opposite functions of GSN and OAS2 on colorectal cancer metastasis, mediating perineural and lymphovascular invasion, respectively[J]. PLoS One, 2018,13(8): e202856.

13. Mao D, Zhang Z, Zhao X, Dong X. Autophagy-related genes prognosis signature as potential predictive markers for immunotherapy in hepatocellular carcinoma[J]. PeerJ, 2020,8: e8383.

14. Qureshi-Baig K, Kuhn D, Viry E, Pozdeev V I, Schmitz M, Rodriguez F, et al. Hypoxia-induced autophagy drives colorectal cancer initiation and progression by activating the PRKC/PKC-EZR (ezrin) pathway[J]. Autophagy, 2020,16(8):1436-1452.

15. Kagaya M, Kaneko S, Ohno H, Inamura K, Kobayashi K. Cloning and characterization of the 5'flanking region of human cytokeratin 19 gene in human cholangiocarcinoma cell line[J]. J Hepatol, 2001,35(4):504-511.

16. Kang M R, Kim M S, Oh J E, Kim Y R, Song S Y, KimS S, et al. Frameshift mutations of autophagyrelated genes ATG2B, ATG5, ATG9B and ATG12 in gastric and colorectal cancers with microsatellite instability[J]. J Pathol, 2009,217(5):702-706.

17. Li L, Goedegebuure S P, Gillanders W E. Preclinical and clinical development of neoantigen vaccines[J]. Ann Oncol, 2017,28(suppl 12): i11-i17.

18. Peng $M$, Mo $Y$, Wang $Y$, Wu P, Zhang Y, Xiong F, et al. Neoantigen vaccine: an emerging tumor immunotherapy[J]. Mol Cancer, 2019,18(1):128. 
19. Ott P A, Hu Z, Keskin D B, Seaman M, Buchbinder E I, YoonC H, et al. An immunogenic personal neoantigen vaccine for patients with melanoma[J]. Nature, 2017,547(7662):217-221.

20. Carreno B M, Magrini V, Becker-Hapak M, Kaabinejadian S, Hundal J, Petti A A, et al. Cancer immunotherapy. A dendritic cell vaccine increases the breadth and diversity of melanoma neoantigen-specific T cells[J]. Science, 2015,348(6236):803-808.

21. Harari A, Graciotti M, Bassani-Sternberg M, Kandalaft L E. Antitumour dendritic cell vaccination in a priming and boosting approach[J]. Nat Rev Drug Discov, 2020,19(9):635-652.

22. He K, Liu S, Xia Y, Xu J, Liu F, Xiao J, et al. CXCL12 and IL7R as Novel Therapeutic Targets for Liver Hepatocellular Carcinoma Are Correlated with Somatic Mutations and the Tumor Immunological Microenvironment[J]. Front Oncol, 2020,10:574853.

23. Prieto J, Melero I, Sangro B. Immunological landscape and immunotherapy of hepatocellular carcinoma[J]. Nat Rev Gastroenterol Hepatol, 2015,12(12):681-700.

24. Jardim D L, Goodman A, de Melo G D, Kurzrock R. The Challenges of Tumor Mutational Burden as an Immunotherapy Biomarker[J]. Cancer Cell, 2021,39(2):154-173.

25. Petrelli F, Ghidini M, Ghidini A, Tomasello G. Outcomes Following Immune Checkpoint Inhibitor Treatment of Patients with Microsatellite Instability-High Cancers: A Systematic Review and Metaanalysis[J]. JAMA Oncol, 2020,6(7):1068-1071.

26. Roberti M P, Yonekura S, Duong C, Benoist S, Scoazec J Y, Dartigues P, et al. Chemotherapy-induced ileal crypt apoptosis and the ileal microbiome shape immunosurveillance and prognosis of proximal colon cancer[J]. Nat Med, 2020,26(6):919-931.

27. Park S L, Buzzai A, Rautela J, Effern M, McBain N, Wagner T, et al. Tissue-resident memory CD8(+) T cells promote melanoma-immune equilibrium in skin[J]. Nature, 2019,565(7739):366-371.

28. Oliva M, Spreafico A, Taberna M, Alemany L,Coburn B, Mesia R, et al. Immune biomarkers of response to immune-checkpoint inhibitors in head and neck squamous cell carcinoma[J]. Ann Oncol, 2019,30(1):57-67.

29. Mahmood N, Rabbani S A. DNA Methylation Readers and Cancer: Mechanistic and Therapeutic Applications[J]. Front Oncol, 2019,9:489.

30. Tse J, Jenkins L J, Chionh F, Mariadason J M. Aberrant DNA Methylation in Colorectal Cancer: What Should We Target ? [J]. Trends Cancer, 2017,3(10):698-712.

31. Gyorffy B, Bottai G, Fleischer T, Munkacsy G, Budczies J, Paladini L, et al. Aberrant DNA methylation impacts gene expression and prognosis in breast cancer subtypes[J]. Int J Cancer, 2016,138(1):8797.

32. Koch A, Joosten S C, Feng Z, de Ruijter T C, Draht M X, Melotte V, et al. Analysis of DNA methylation in cancer: location revisited[J]. Nat Rev Clin Oncol, 2018,15(7):459-466.

33. Fu R, Ding Y, Luo J, Huang K M, Tang X J, Li D S, et al. Ten-eleven translocation 1 regulates methylation of autophagy-related genes in human glioma[J]. Neuroreport, 2018,29(9):731-738. 
34. Chen Z, Gong L, Zhang P, Li Y, Liu B, Zhang L, et al. Epigenetic Down-Regulation of Sirt 1 via DNA Methylation and Oxidative Stress Signaling Contributes to the Gestational Diabetes Mellitus-Induced Fetal Programming of Heart Ischemia-Sensitive Phenotype in Late Life[J]. Int J Biol Sci, 2019,15(6):1240-1251.

35. Gonzalez-Rodriguez P, Cheray M, Füllgrabe J, Salli M, Engskog-Vlachos P, Keane L, et al. The DNA methyltransferase DNMT3A contributes to autophagy long-term memory[J]. Autophagy, 2020:1-19.

36. Jiang L, Zhao X H, Mao Y L, WangJ F, Zheng H J, You Q S. Long non-coding RNA RP11-468E2.5 curtails colorectal cancer cell proliferation and stimulates apoptosis via the JAK/STAT signaling pathway by targeting STAT5 and STAT6[J]. J Exp Clin Cancer Res, 2019,38(1):465.

37. Dong Z, Chen Y, Yang C, Zhang M, Chen A, Yang J, et al. STAT gene family mRNA expression and prognostic value in hepatocellular carcinoma[J]. Onco Targets Ther, 2019,12:7175-7191.

38. Gu C, Cai J, Xu Z, Zhang M, Chen A, Yang J, et al. MiR-532-3p suppresses colorectal cancer progression by disrupting the ETS1/TGM2 axis-mediated Wnt/ $\beta$-catenin signaling[J]. Cell Death Dis, 2019,10(10):739.

39. Chen Y, Peng C, Chen J, Chen D, Yang B, He B, et al. WTAP facilitates progression of hepatocellular carcinoma via m6A-HuR-dependent epigenetic silencing of ETS1[J]. Mol Cancer, 2019,18(1):127.

40. Chen C, Aihemaiti M, Zhang X, Qu H, Sun Q L, He Q S, et al. Downregulation of histone demethylase JMJD1C inhibits colorectal cancer metastasis through targeting ATF2[J]. Am J Cancer Res, 2018,8(5):852-865.

\section{Figures}




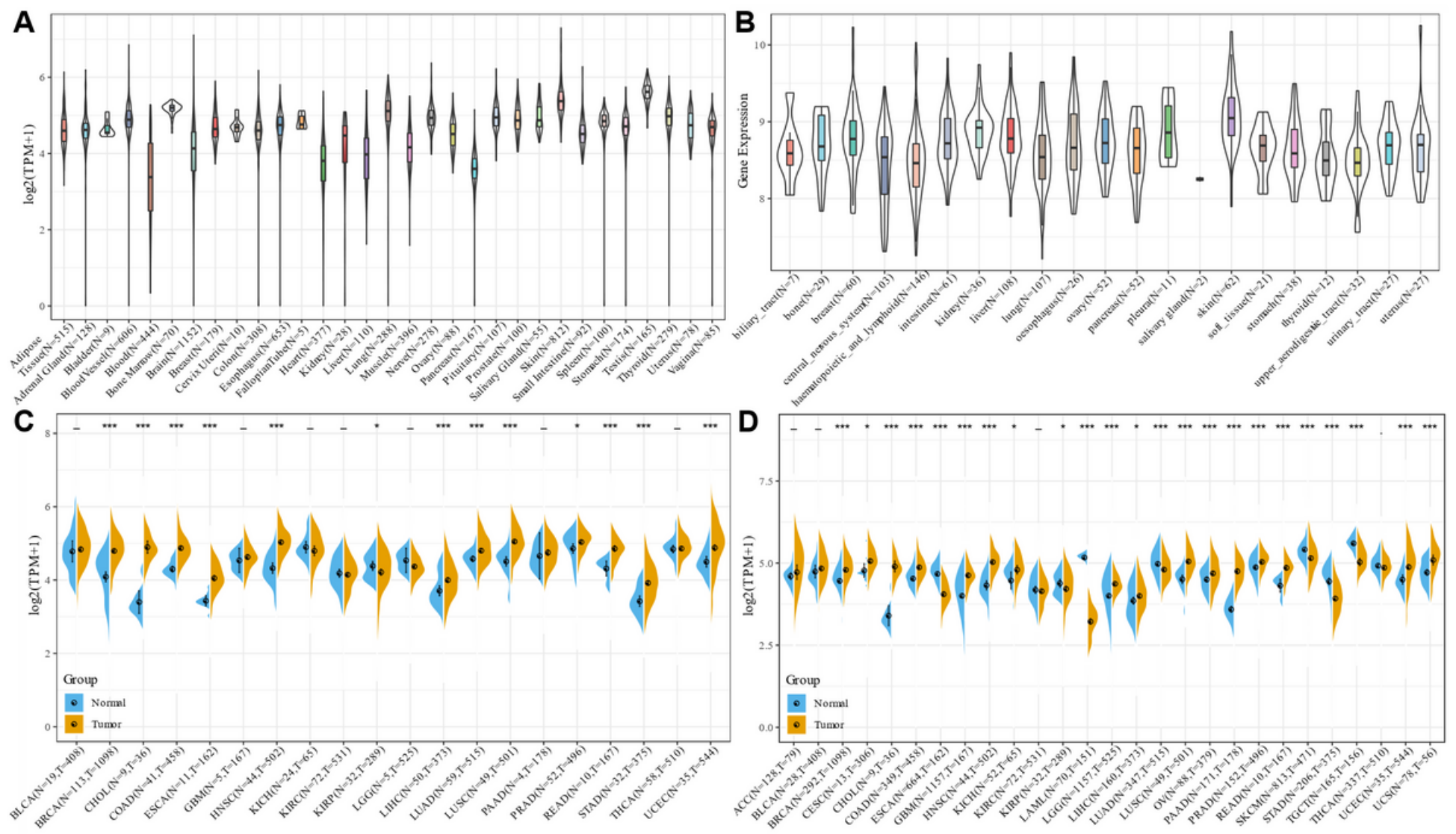

Figure 1

The expression level of ATG101 in different cancer patients. (A) The expression of ATG101 in 31 normal tissues from the GTEx database. (B) The expression of ATG101 in 21 types of malignant cancer cells from the CCLE database. (C) The expression of ATG101 between 20 types of malignant tumour tissues and normal tissues from the TCGA database. (D) The expression of ATG101 in 27 types of malignant tumour tissues from the TCGA database and the corresponding normal tissues from the GTEx database. ${ }^{\star} \mathrm{P}<0.05 .{ }^{* \star \mathrm{P}}<0.01$. ${ }^{* *} \mathrm{P}<0.001$. 

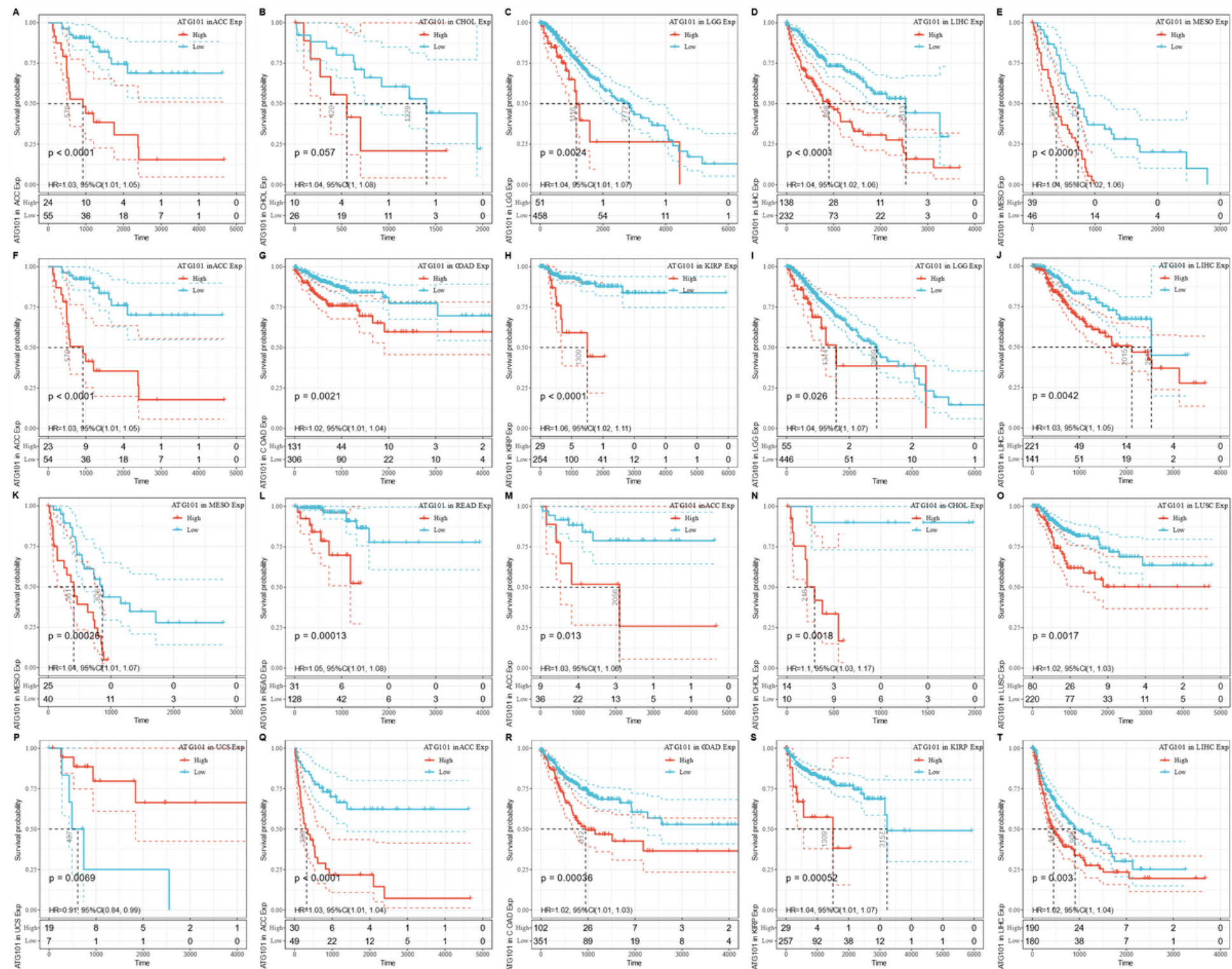

Figure 2

The relationship between the expression of ATG101 and the prognosis of patients with 33 types of malignant tumours from the TCGA database. (A-E) Overall survival. (F-L) Disease-specific survival. (M-P) Disease-free survival. (Q-T) Progression-free survival. 
A
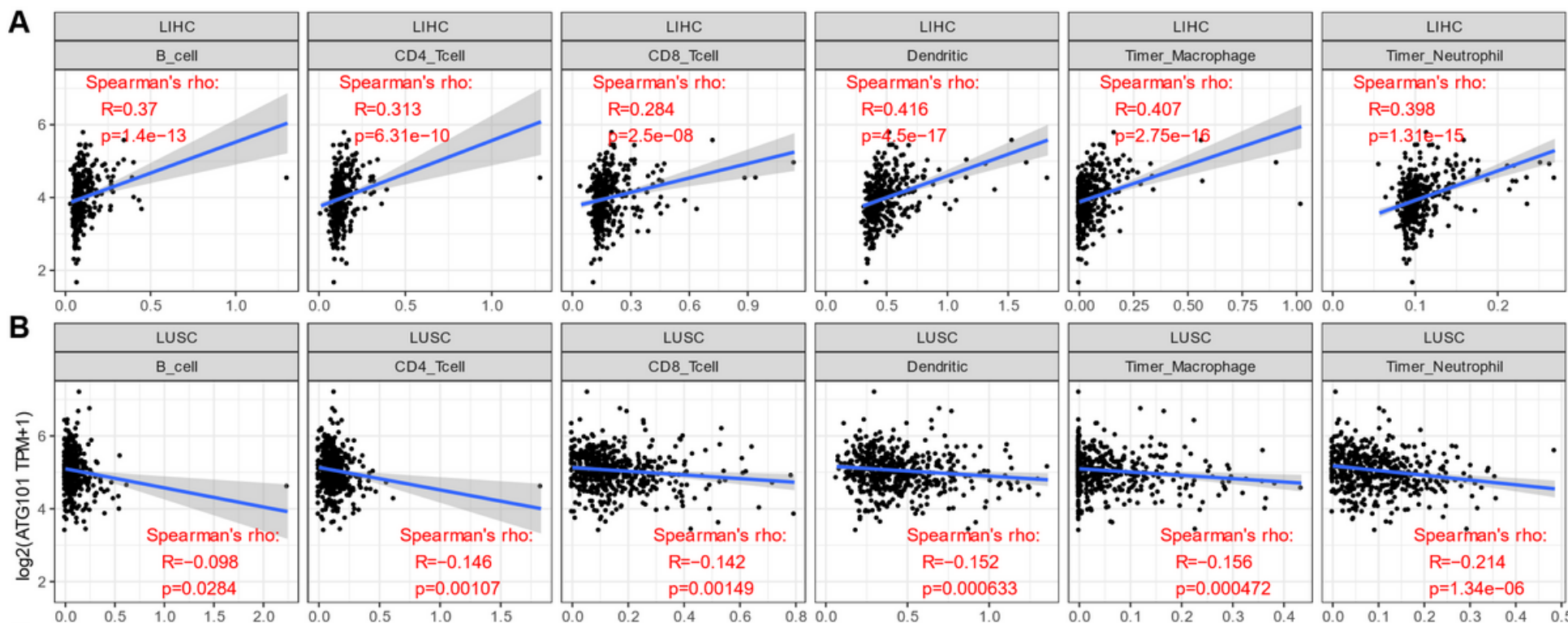

C
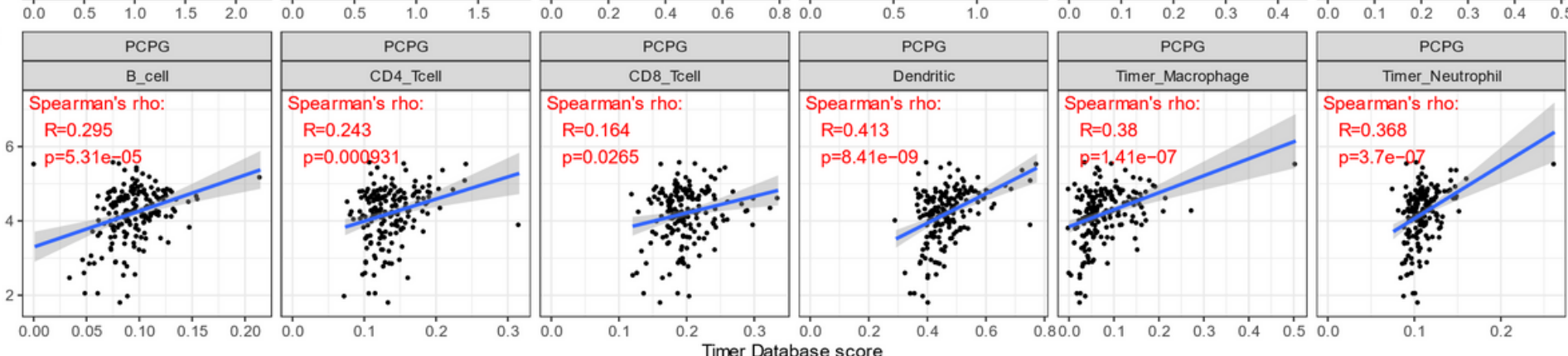

\section{Figure 3}

The relationship between the expression level of ATG101 and the infiltration level of six kinds of immune cells in patients with malignant tumours. (A, B, C) The three tumours, LIHC, LUSC and PCPG, respectively, with the strongest correlation with immune cell infiltration. 

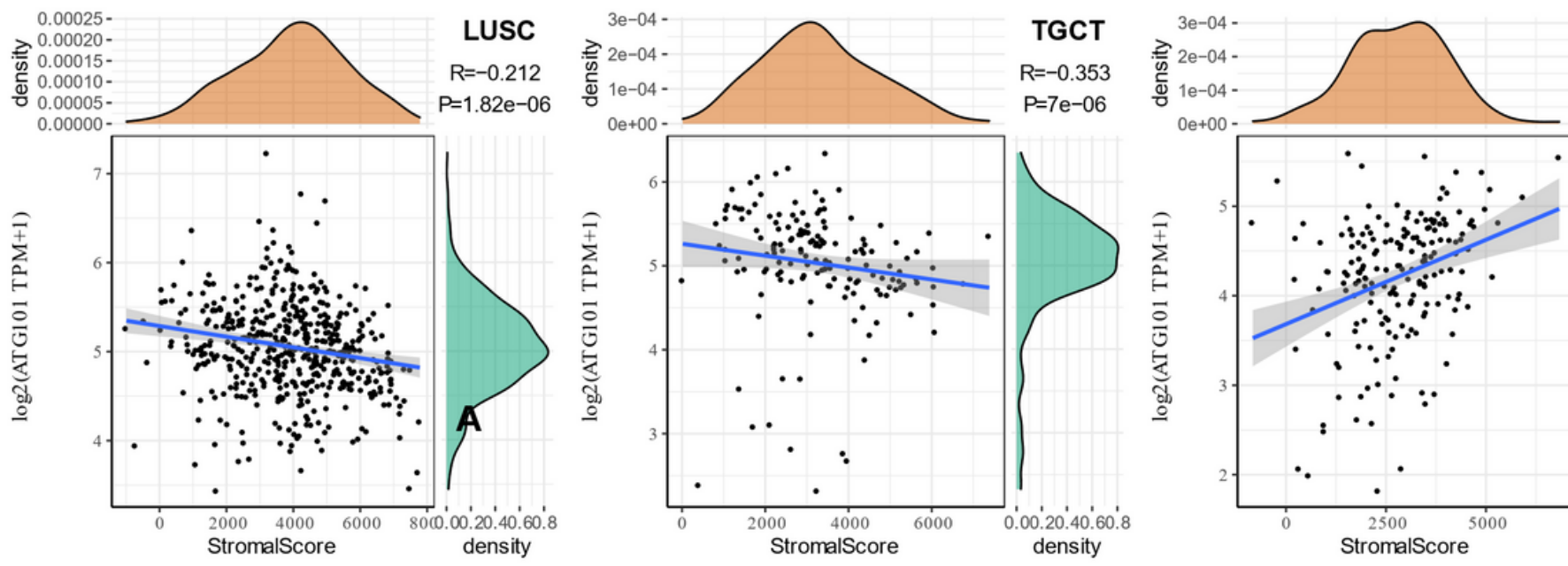

PCPG

$R=0.315$ $P=1.55 e-05$
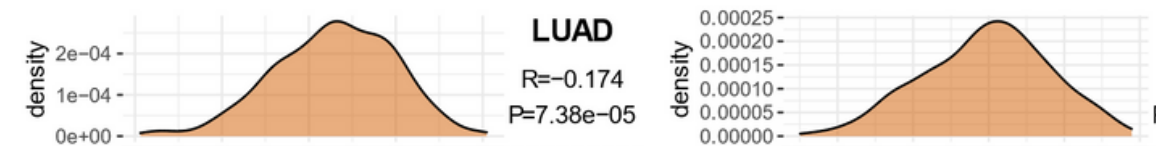

LUSC
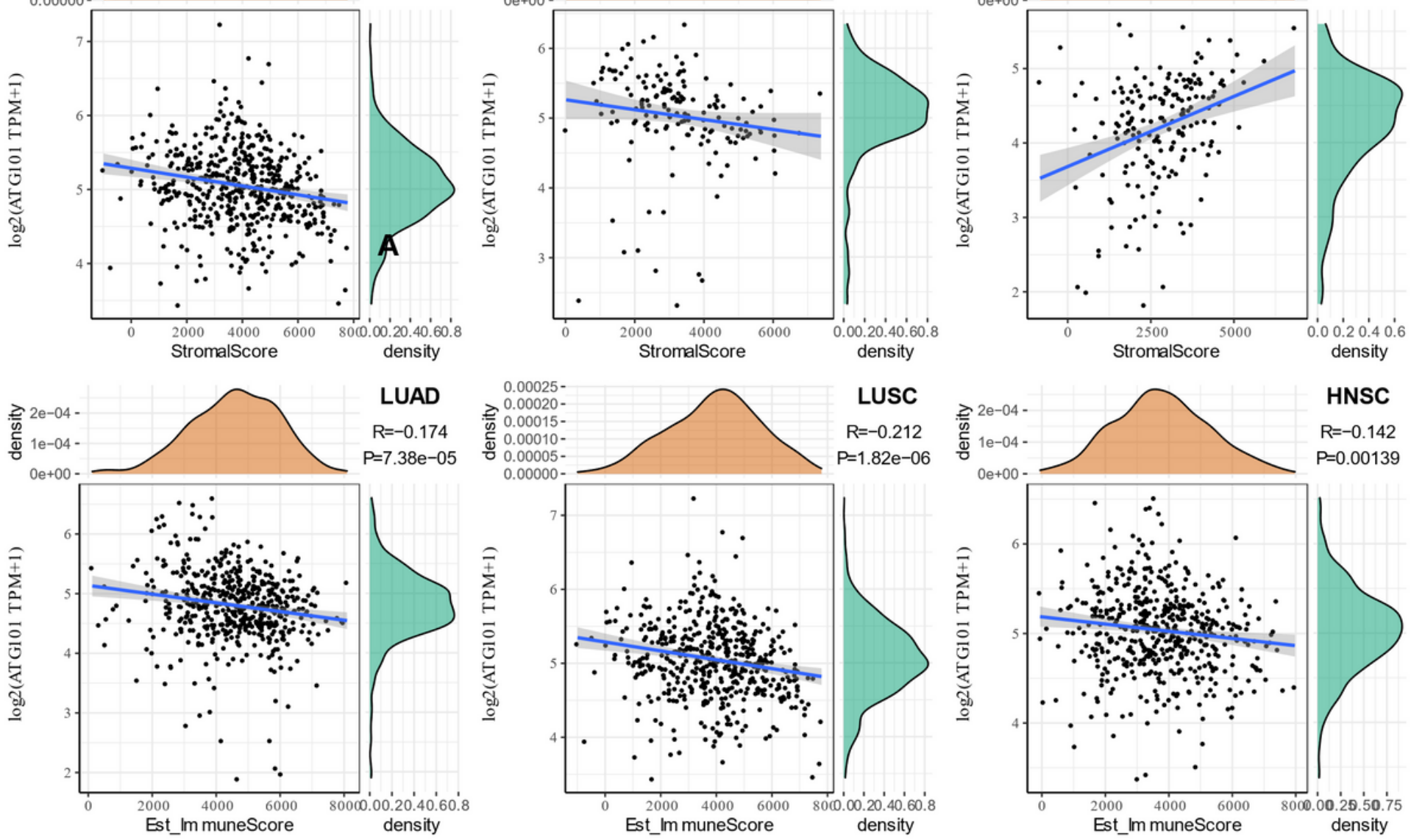

$\mathrm{R}=-0.212$

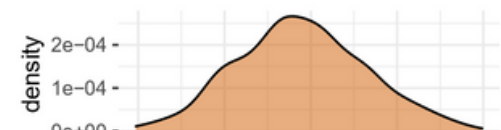

HNSC

$R=-0.142$ $P=0.00139$
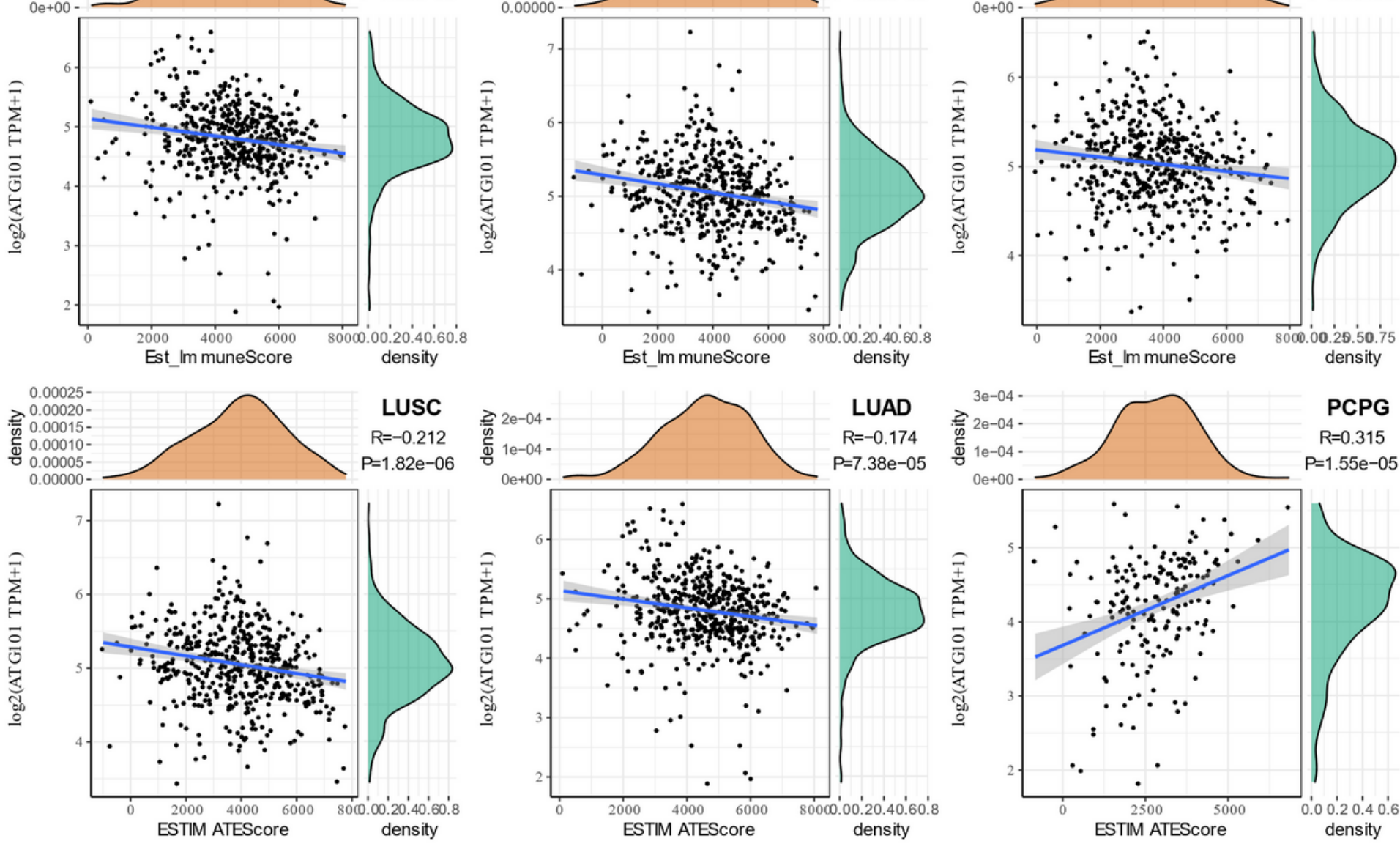

PCPG

$R=0.315$ $P=1.55 e-05$
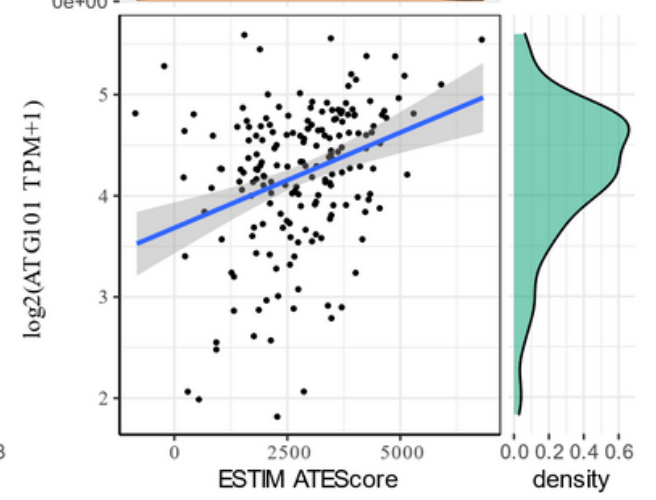

Figure 4

The relationship between the algorithm score in cancer patients and the expression level of ATG101. The third highest correlations with the stromal score, immune score and ESTIMATE score. 
A

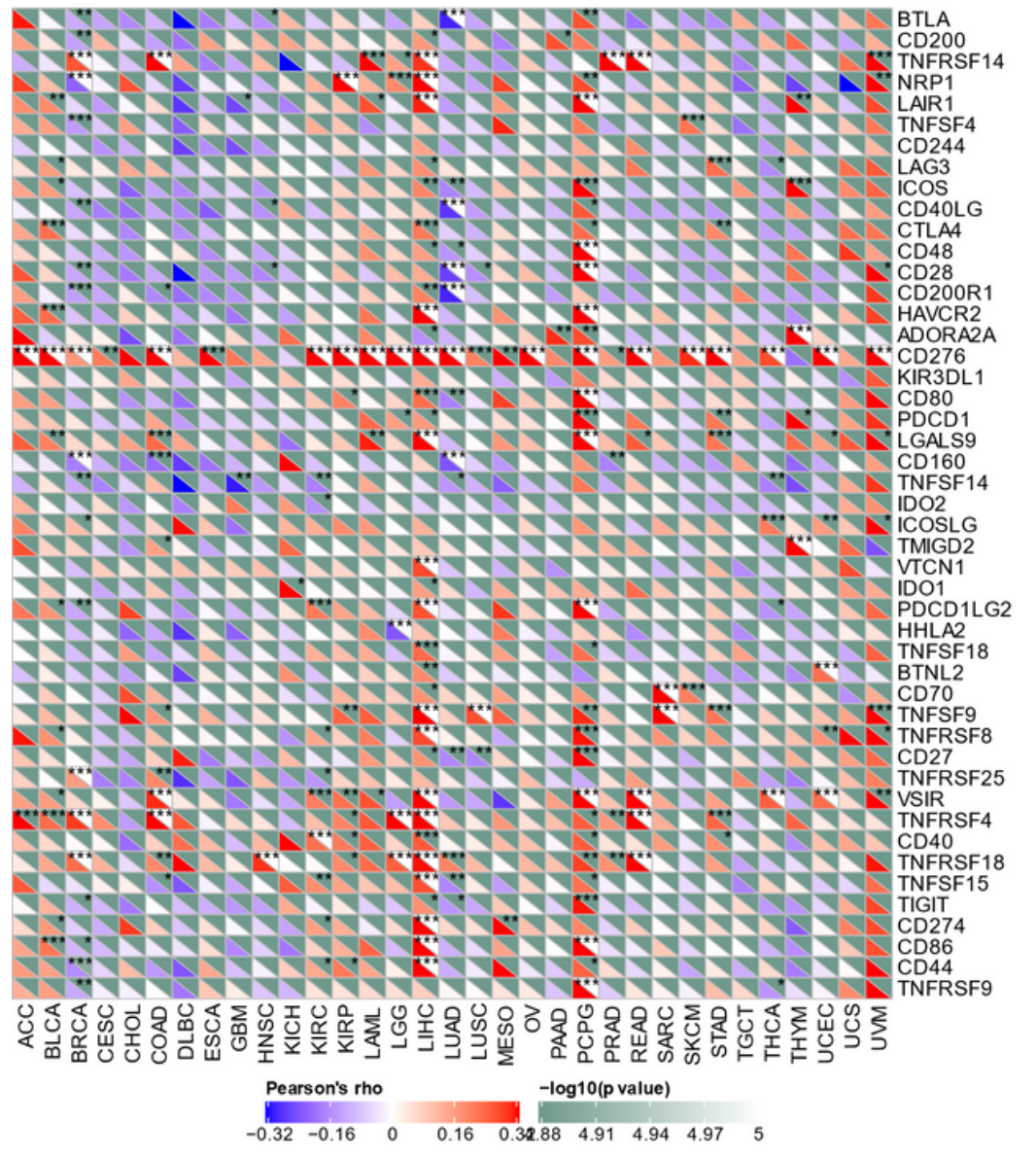

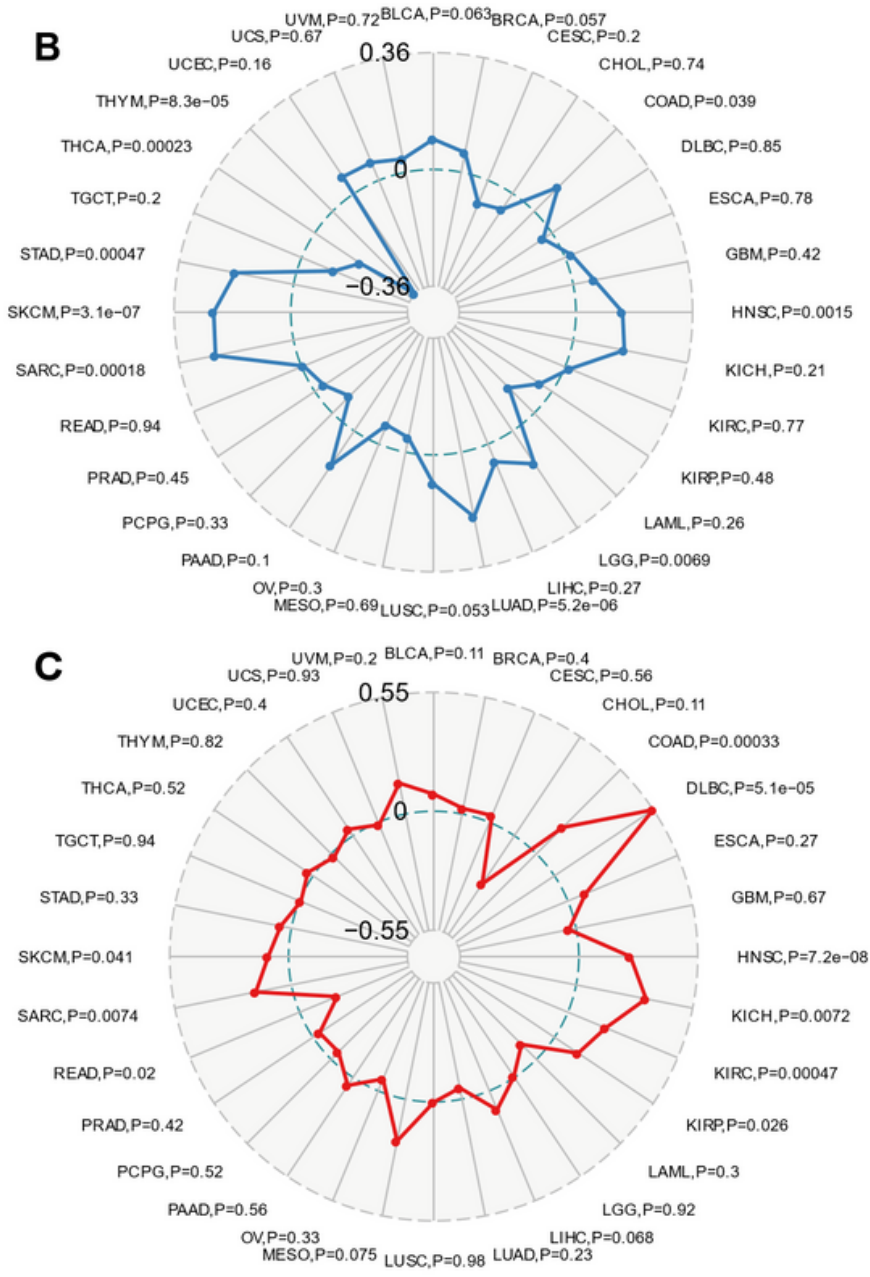

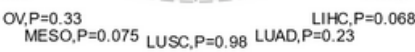

\section{Figure 5}

The relationship between ATG101 expression and immunoreactivity. (A) The correlation between immune checkpoint gene expression and ATG101 expression level. ${ }^{\star} P<0.05$. ${ }^{\star} P P<0.01$. ${ }^{* \star} P<0.001$. (B) The relationship between ATG101 expression level and TMB. (C) ATG101 expression level and microsatellite instability (MSI) in tumour patients. 


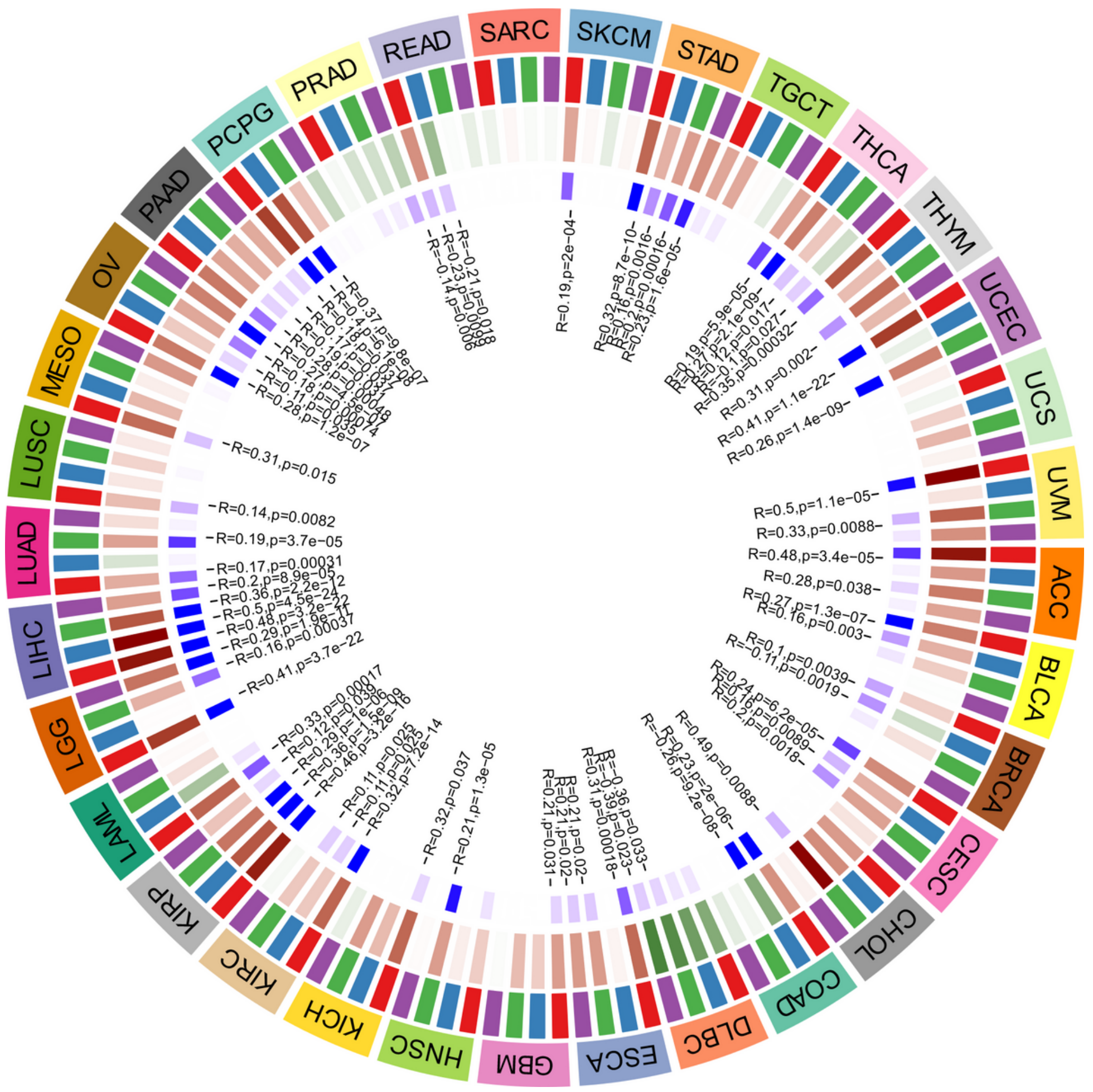

Figure 6

The relationship between 4 methyltransferases and the expression level of ATG101. 

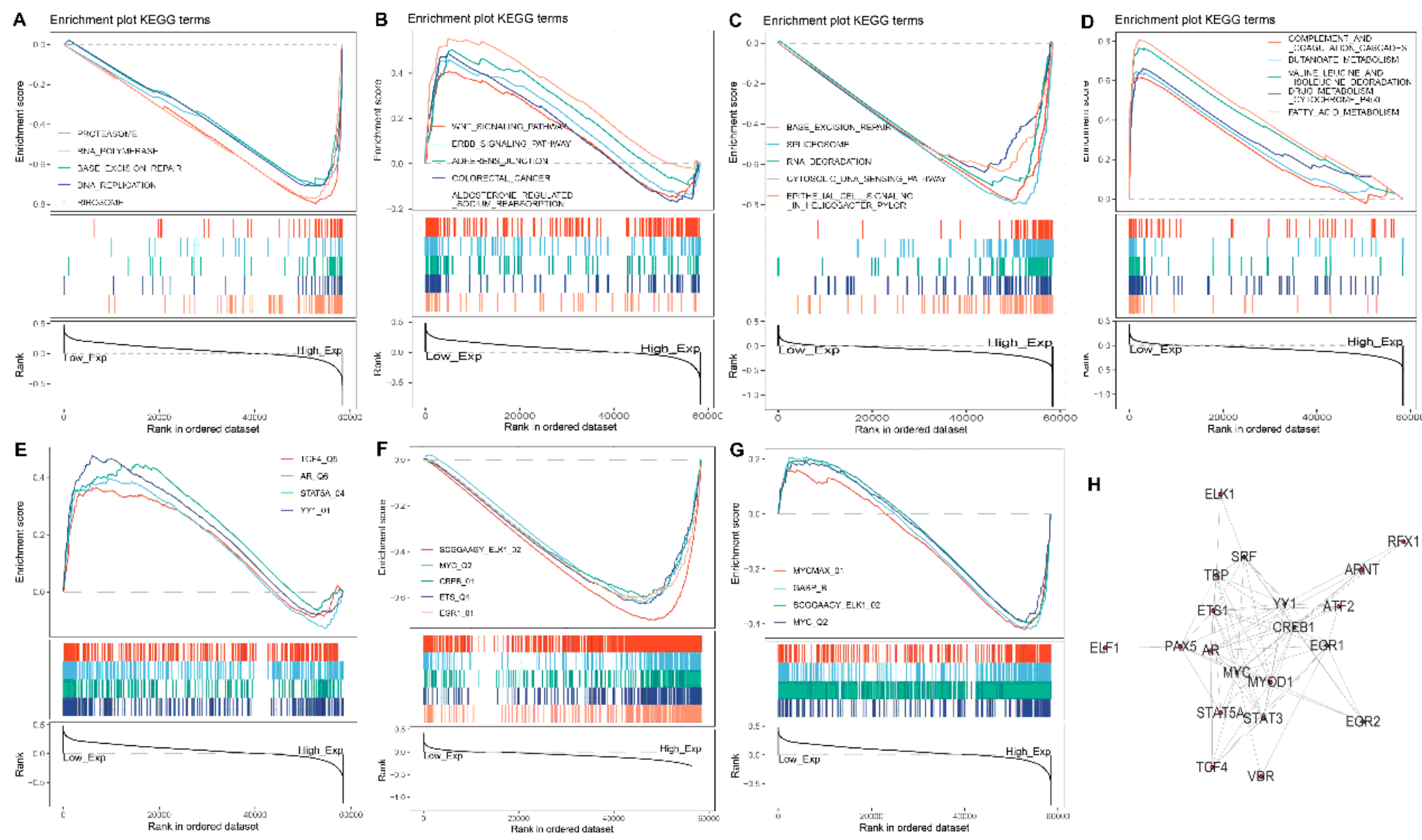

H
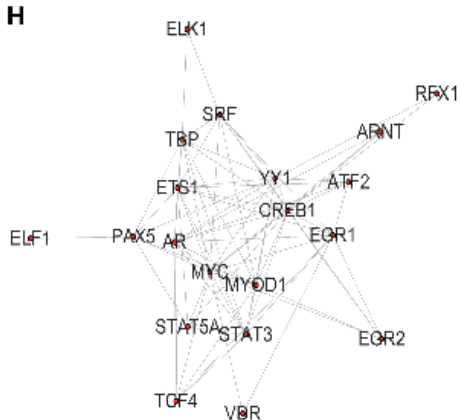

\section{Figure 7}

KEGG pathway analysis of ATG101 by GSEA in COAD and LIHC (A, B) The pathways are negatively and positively correlated with ATG101 expression in COAD. (C, D) The pathways are negatively and positively correlated with ATG101 expression in LIHC. $(E, F)$ The transcription factors are positively and negatively correlated with ATG101 expression in COAD. (G) The transcription factors are correlated with ATG101 expression in LIHC. (H) Twenty-one common genes related to ATG101, COAD and LIHC.

\section{Supplementary Files}

This is a list of supplementary files associated with this preprint. Click to download.

- S1.png

- S2.png

- S3.png

- S4.png 